\title{
Expression and Regulation of L-Selectin on Eosinophils from Human Adults and Neonates ${ }^{1}$
}

\author{
JEFFREY B. SMITH, RAJU D. KUNJUMMEN, TAKASHI K. KISHIMOTO, AND \\ DONALD C. ANDERSON
}

\begin{abstract}
Department of Pediatrics, UCLA School of Medicine, Los Angeles, California 90024 [J.B.S., R.D.K.]; Boehringer Ingelheim Pharmaceuticals, Inc., Ridgefield, Connecticut 06877 [T.K.K.]; and Department of Pediatrics, Baylor College of Medicine, Houston, Texas 77030 [D.C.A.]
\end{abstract}

\begin{abstract}
L-Selectin, previously known as LEC.CAM1, LECAM-1, LAM1, and as the MEL-14, Leu-8, TQ1, and DREG-56 antigens, is a leukocyte membrane protein that participates in adhesion to endothelium. We studied its expression on eosinophils using flow cytometry and the MAb Dreg-56 and Leu-8. Unstimulated peripheral blood eosinophils from healthy adults expressed about one third the level of $\mathrm{L}$-selectin as neutrophils (mean \pm SD specific fluorescence: $20.9 \pm 3.2$ versus $54.5 \pm 8.4, p=0.0001, n$ $=18$ ). After stimulation with A23187, L-selectin expression on eosinophils was rapidly lost. This was temporally correlated with increased expression of Mac-1 (CD11b/ CD18); the kinetics on eosinophils and neutrophils were similar. Eosinophil expression of $\mathrm{L}$-selectin decreased modestly after stimulation with platelet activating factor, but was minimally affected by $\mathbf{N}$-formyl-methionyl-leucylphenylalanine, leukotriene B4, or C5a compared with their effects on neutrophils. Eosinophils from cord blood of healthy neonates born at term expressed less $L$-selectin than adult eosinophils $(10.4 \pm 3.8$ versus $19.4 \pm 2.7, p=$ $0.0001, n=9$ ); the relative reduction was the same as on cord blood neutrophils $(36.4 \pm 8.2$ versus $55.5 \pm 4.8, p=$ $0.0001, n=9$ ). Relative to baseline expression, the responses of neonatal and adult cells to stimulation did not differ. We conclude that neonatal eosinophils have abnormalities in L-selectin expression similar to neonatal neutrophils and suggest that decreased expression of L-selectin and a diminished responsiveness to direct stimulation with chemotactic factors are possible mechanisms that may limit the exudation of eosinophils. (Pediatr Res 32: 465471, 1992)
\end{abstract}

\section{Abbreviations}

FMLP, N-formyl-methionyl-leucyl-phenylalanine

LTB4, leukotriene B4

PAF, platelet activating factor

PE, phycoerythrin

HBSS, Hanks' balanced salt solution

FBS, fetal bovine serum

Better understanding of the molecular mechanisms that regu-

Received January 28, 1992; accepted May 13, 1992.

Correspondence and reprint requests: Jeffrey B. Smith, M.D., Ph.D., Pediatrics/ Neonatology, UCLA Center for the Health Sciences, 10833 Le Conte Ave., Los Angeles, CA 90024.

Supported in part by funds provided by the Committee on Research of the Academic Senate of the Los Angeles Division of the University of California, and by Cancer Center Core Grant CA-16042.

Presented in part at the Annual Meeting of the American Pediatric Society and the Society for Pediatric Research, May 1991. late eosinophil trafficking could lead to important advances in the prevention and treatment of asthma, acute lung injury, and other conditions in which eosinophil-mediated tissue injury may play an important role (1-3). The existence of abnormalities in eosinophil trafficking in the neonatal period is suggested by several longstanding clinical observations. Neonatal inflammatory exudates often contain an increased proportion of eosinophils (4-6), and an infiltrate of eosinophils is characteristic of erythema toxicum, a self-limited rash that occurs in as many as $70 \%$ of normal neonates (7). It is also well known that a majority of premature infants develop significant eosinophilia during the first several weeks of postnatal life $(8,9)$. We recently reported that, in fetuses with $\mathrm{Rh}$ disease studied at 20-26 wk gestation by in utero umbilical cord sampling, $60 \%$ of circulating granulocytes were eosinophils (10). Even in the healthy fetus, eosinophils average 10 to $20 \%$ of granulocytes at that stage of gestation (11). The mechanisms underlying these phenomena are not known.

Adhesion to endothelium via the specific interaction of cellsurface adhesion molecules is a crucial step in the process of leukocyte emigration from the bloodstream to the tissues (12, 13). Like neutrophils, eosinophils express the leukocyte integrins (CD18 family), and adhesion of eosinophils to endothelium via CD18-dependent mechanisms has been demonstrated (14-17). The importance of this family of adhesion receptors is exemplified by the profound defects of neutrophil exudation shown by patients with the severe form of the leukocyte adhesion deficiency syndrome, in which the CD18 molecules are congenitally absent (18). However, CD18-independent adhesion mechanisms also exist and may be of even greater importance for eosinophils than neutrophils, inasmuch as eosinophils of patients with the leukocyte adhesion deficiency syndrome are able to migrate into infected tissues (19). Like neutrophils, eosinophils bind to endothelial ELAM-1 (20-22). Unlike neutrophils, eosinophils express VLA-4, which binds to endothelial VCAM-1 (21-24). Another potential mechanism for CD18-independent adherence of eosinophils involves a leukocyte adhesion molecule recently designated L-selectin (25).

The selectins are a family of integral membrane proteins characterized by the presence of lectin-, epidermal growth factor, and complement-binding domains $(25,26)$. L-selectin was previously known as LEC.CAM-1, LECAM-1, LAM 1, and as the MEL-14, Leu-8, TQ1, and Dreg-56 antigens (25, 27-31). It is involved in lymphocyte homing to peripheral lymph nodes and in the adhesion of neutrophils to inflamed endothelium (31, 32). Stimulation of neutrophils with chemotactic factors simultaneously produces a rapid decrease in expression of L-selectin together with an increase in expression of the leukocyte integrin Mac-1 (CDI1b/CD18) (33, 34). Although closely coordinated, the mechanisms of these changes are different. The decrease in $\mathrm{L}$-selectin is due to shedding from the cell surface of a fragment that is about $5 \mathrm{kD}$ smaller than the cell-associated form ( $100 \mathrm{kD}$ on neutrophils) $(33,35)$, whereas the increase in Mac-1 (CD1 lb/ 
CD18) expression occurs by translocation of molecules from an intracellular pool to the plasma membrane (36). In addition to these changes in quantitative surface expression, cell stimulation also results in functional activation of these molecules $(36,37)$. The roles of these molecules in neutrophil adhesion appear to be distinct. It has been proposed that L-selectin is primarily involved in the initial adhesion of neutrophils to inflamed endothelium, especially under conditions of flow where CD18-dependent mechanisms are not significant, whereas Mac-1 is involved in subsequently strengthening the adhesion and in transendothelial migration (32). Shedding of L-selectin may be required for subsequent steps in migration to occur (38). Neonatal neutrophils have diminished expression of L-selectin compared to neutrophils from adults, and this correlates with diminished adhesion of neonatal neutrophils to IL-1-stimulated endothelium in conditions of flow (39).

The purpose of this study was to investigate the expression and regulation of $\mathrm{L}$-selectin on eosinophils from adults and neonates. L-Selectin is known to be expressed on eosinophils from adults $(27,40)$, but regulation of its expression in response to stimulation has not been studied on either adult or neonatal eosinophils. Cell-specific responses to chemotactic factors, together with cell-specific patterns of expression of different types of adhesion molecules, may be an important mechanism for selective regulation of the emigration of different classes of leukocytes $(34,40)$. We recently reported dramatic differences between eosinophils and neutrophils in Mac-1 up-regulation in response to stimulation with FMLP, C5a, PAF, LTB4 (41). We also showed that up-regulation of Mac- 1 on neonatal eosinophils is significantly diminished compared to eosinophils from adults (41), similar to the defect previously demonstrated in neonatal neutrophils $(10,42,43)$. We hypothesized that, if coordinate upregulation of Mac-1 and down-regulation of $\mathrm{L}$-selectin is a general feature of the regulation of the receptors on granulocytes, then changes in eosinophil L-selectin expression would follow the same pattern of responsiveness to different stimulating factors as shown for Mac-1. We also hypothesized that L-selectin expression on neonatal eosinophils would be diminished compared to eosinophils from adults.

\section{MATERIALS AND METHODS}

Subjects. Blood specimens were drawn from a peripheral vein of healthy adult volunteers or from the placental side of the clamped umbilical cord immediately after uncomplicated vaginal or cesarean deliveries of healthy infants at term gestation (38-41 wk). The specimens were obtained, with appropriate consent, in accordance with an approved institutional protocol.

Materials. The Dreg-56 antibody (IgGl isotype) was produced and characterized as an anti-L-selectin antibody as previously described (31). PE-conjugated anti-Leu-8 (IgG2a) (anti-L-selectin), PE-conjugated anti-Leu-15 (IgG2a) (CD11b, anti-Mac-1 $\alpha$ chain), FITC-conjugated anti-Leu-1 la (IgG1) (CD16, anti$\mathrm{Fc}_{\gamma} \mathrm{R}_{\mathrm{III}}$ ), and control MAb [unconjugated and FITC-conjugated mouse IgGI (clone X40) and PE-conjugated mouse IgG2a (clone X39)] were obtained from Becton Dickinson (Mountain View, CA). Human Ig-absorbed PE-conjugated goat anti-mouse $F\left(a b^{\prime}\right)_{2}$ antibodies (Tago, Inc., Burlingame, CA) were used for secondstep labeling of Dreg-56 and its control. PE-conjugated beads from Flow Cytometry Standards Corporation (Research Triangle Park, NC) were used in each experiment as a fluorescence standard.

HBSS without bicarbonate or phenol red and PBS were obtained from GIBCO BRL (Grand Island, NY). HBSS was supplemented with $2 \%$ by volume heat-inactivated FBS (HBSSFBS), adjusted to $\mathrm{pH}$ 7.35-7.40, and maintained sterile until use. HBSS-FBS without $\mathrm{Ca}$ and $\mathrm{Mg}$ was the buffer in all experiments except as specified. For eosinophil purification, the Dynal MPC1 magnetic particle concentrator and $4.5-\mu \mathrm{m}$ polystyrene magnetic beads (Dynabeads M-450) coated with goat anti-mouse IgG were obtained from Robbins Scientific (Mountain View, CA).

Calcium ionophore A23187, FMLP, human recombinant C5a, PAF, LTB4, zymosan, histamine, and the eosinophil chemotactic factor of anaphylaxis tetrapeptides Ala-Gly-Ser-Glu and ValGly-Ser-Glu were obtained from Sigma Chemical Co. (St. Louis, MO). Colloidal silica particles coated with polyvinyl pyrrolidone (Percoll) were purchased from Pharmacia (Piscataway, NJ).

Cell preparation. To minimize the possibility that L-selectin could be lost from the cell surface by inadvertent activation of the cells during in vitro procedures, EDTA-anticoagulated blood specimens were placed on ice immediately after collection, and all purification and labeling procedures were carried out at 0 $4^{\circ} \mathrm{C}$. For experiments using whole blood leukocytes, erythrocytes were removed by two cycles of hypotonic lysis and washing in HBSS-FBS, and the cell suspension was used with no further processing. For experiments using mixtures of eosinophils and neutrophils, two-layer discontinuous gradients of Percoll in PBS were prepared as described by Roberts and Gallin (44). Whole blood was diluted with isotonic PBS (1:1 for adult specimens or 1:2 for neonatal specimens with a total leukocyte count $\geq 12 \times$ $10^{6} / \mathrm{mL}$ ) and made slightly hypertonic by the addition of $10 \mu \mathrm{L}$ of $10 \times$-concentrated PBS per $\mathrm{mL}$ of the diluted blood. The cells were not exposed to FMLP. Up to $6 \mathrm{~mL}$ of cold, diluted blood was overlaid on gradients of $65 \%$ and $70 \%$ Percoll $(3 \mathrm{~mL}$ in each layer) in a $15-\mathrm{mL}$ conical tube and centrifuged at $4^{\circ} \mathrm{C}$ for $20 \mathrm{~min}$ at $250 \times g$. The plasma layer and the cells at the upper interface were discarded, and the cells at the interface of the two Percoll layers were collected. If necessary, residual erythrocytes were removed by one cycle of hypotonic lysis after the cells were washed in HBSS-FBS to remove Percoll. For brevity, we refer to these preparations as granulocyte suspensions, but it should be noted that they do not include basophils, which remain with the mononuclear cells on Percoll gradients. Differential counts of Wright-stained smears of whole blood and of cytocentrifuge preparations of the whole blood leukocyte or granulocyte suspensions were made for each specimen.

Eosinophil purification. A modification of the procedure described by Hansel et al. (45) was used. Briefly, granulocytes prepared on a cold Percoll gradient as described above were resuspended in $200 \mu \mathrm{L}$ of buffer containing $0.1 \% \mathrm{Na}$ azide and incubated with anti-Leu- 1 la ( $40 \mu \mathrm{L}$ per $10^{6}$ cells) for $30 \mathrm{~min}$ on ice. The cells were then washed to remove unbound antibody, resuspended in a minimal volume of buffer $\left(40 \mu \mathrm{L}\right.$ for $10^{6}$ cells) in a $1.5 \mathrm{~mL}$ microcentrifuge tube, mixed with an aliquot of goat anti-mouse IgG-coated magnetic beads $\left(25 \mu \mathrm{L}\right.$ containing $10^{7}$ beads for $10^{6}$ cells), and incubated at $4^{\circ} \mathrm{C}$ for $15 \mathrm{~min}$ with continuous rotation. The tube was then placed in a magnet (the Dynal MPC1) to retain the beads and bound cells, and the supernatant and unbound cells were removed by washing. After resuspension in $40 \mu \mathrm{L}$ of buffer, the unbound cells were incubated with a second aliquot of the magnetic beads and the separation was repeated. The purity of the preparation was verified by a differential count of a Wright-stained cytocentrifuge slide. Preparations containing $>98 \%$ eosinophils were obtained routinely.

Cell stimulation. Incubation of the cells with stimulating factors was performed in a shaking water bath at $37^{\circ} \mathrm{C}$. HBSS-FBS with $\mathrm{Ca}$ and $\mathrm{Mg}$ was used in this step. Parallel aliquots kept on ice (cold control) or incubated with buffer alone $\left(37^{\circ} \mathrm{C}\right.$ control) were included in each experiment. In the kinetics experiments, the cells were warmed for $10 \mathrm{~min}$ at $37^{\circ} \mathrm{C}$ before mixing (time zero) with solutions of the stimulating factor in buffer at $37^{\circ} \mathrm{C}$. At intervals, aliquots were removed from the bath, mixed with cold buffer, and placed on ice.

Antibody labeling. HBSS-FBS (without $\mathrm{Ca}$ or $\mathrm{Mg}$ ) with $0.1 \%$ $\mathrm{Na}$ azide was used in the remaining steps of the procedure. In each labeling step, the cells were resuspended in $0.1 \mathrm{~mL}$ of azide buffer, incubated for $30 \mathrm{~min}$ on ice with saturating amounts of antibody (as determined in preliminary experiments), and then 
washed in $2 \mathrm{~mL}$ of azide buffer. Experiments using Dreg-56 required three labeling steps: incubation with Dreg-56 (or mouse IgGI control MAb), then with PE-conjugated goat anti-mouse $\operatorname{IgG~} \mathrm{F}\left(\mathrm{ab}^{\prime}\right)_{2}$, and finally with FITC-conjugated anti-Leu-1 1a. For the PE-conjugated MAb (Leu-8, Leu-15, or mouse IgG2a control), labeling was done in a single step together with the FITCconjugated anti-Leu-11a. After a final wash to remove unbound antibody, the cells were resuspended in $0.3 \mathrm{~mL}$ of azide buffer for analysis.

Flow cytometry and sorting. Analysis of eosinophils and neutrophils in mixed suspensions was performed on a FACScan flow cytometer (Becton Dickinson) as previously described (41). Briefly, red fluorescence (of PE-labeled antibody) was measured using linear amplification and expressed relative to the fluorescence of PE-conjugated beads measured in each experiment. Fluorescence of the PE-conjugated control (nonspecific background) was subtracted to give the net fluorescence corresponding to specific antibody binding. Corrections for spectral overlap (color compensation) were set using labeled and unlabeled neutrophils. Light-scatter gates were used to select the granulocyte region (neutrophils and eosinophils), excluding lymphocytes, monocytes, and basophils (46). Eosinophils (CDi6-dim) and neutrophils (CD16-bright) were distinguished by their well-separated peaks on the CD16 (FITC-Leu 1 la) fluorescence distribution $(41,46)$. For eosinophils, the light-scatter gate was refined by back-gating of the CD16-dim granulocytes as described (41). Cell sorting was performed on a FACStar Plus (Becton Dickinson). Granulocytes or whole blood leukocyte suspensions were incubated with FITC-anti-Leu-1 la, and the populations for sorting were defined as described above. The sorted cells were transferred to glass slides using a cytocentrifuge, then Wrightstained and counted.

Statistics. Mean fluorescence of individual distributions was calculated with the FACScan Research Software, version B (Becton Dickinson). Group statistics were calculated with StatView $512+$ (Brainpower Inc., Calabasas, CA) on a Macintosh computer (Apple Computer, Cupertino, CA), and results were expressed as the group means $\pm S D$ of the individual distribution mean values. Statistical differences were assessed by repeatedmeasures analysis of variance and the paired $t$ test.

\section{RESULTS}

Down-regulation of eosinophil L-selectin. Highly purified, unstimulated eosinophils were isolated from blood by Percoll gradient separation at $4^{\circ} \mathrm{C}$ of a mixture of neutrophils and eosinophils, followed by immunomagnetic removal of the neutrophils using a CD16 MAb (45). Typical fluorescence distributions of $\mathrm{L}-$ selectin on eosinophils purified by this method from peripheral blood of a healthy adult donor are shown in Figure 1. L-Selectin was expressed on more than $95 \%$ of unstimulated, cold eosinophils. Incubation of these cells for $20 \mathrm{~min}$ at $37^{\circ} \mathrm{C}$ in buffer had no effect on their expression of L-selectin, whereas incubation with $0.5 \mu \mathrm{M}$ A23187 resulted in a nearly complete loss of Lselectin expression. In contrast, A23187 produced a large increase in eosinophil Mac-1 expression. Incubation of eosinophils with $1 \mu \mathrm{M}$ FMLP produced a minimal decrease in their expression of $\mathrm{L}$-selectin and a minimal increase in Mac-1 compared to the $37^{\circ} \mathrm{C}$ controls. These reciprocal changes in L-selectin and Mac-1 expression on eosinophils were similar to the behavior previously reported for neutrophils stimulated in vitro or in vivo $(33,38)$. The insensitivity of eosinophils to FMLP is consistent with the lack of a high-affinity receptor for this substance on eosinophils $(44,47,48)$.

L-Selectin expression of unstimulated eosinophils. Because surface expression of L-selectin is affected by cell stimulation, we compared its expression on neutrophils and eosinophils using flow cytometry with a multiparameter gating method that allowed these cells to be distinguished in mixed leukocyte suspensions (41). This ensured that the two cell types were studied

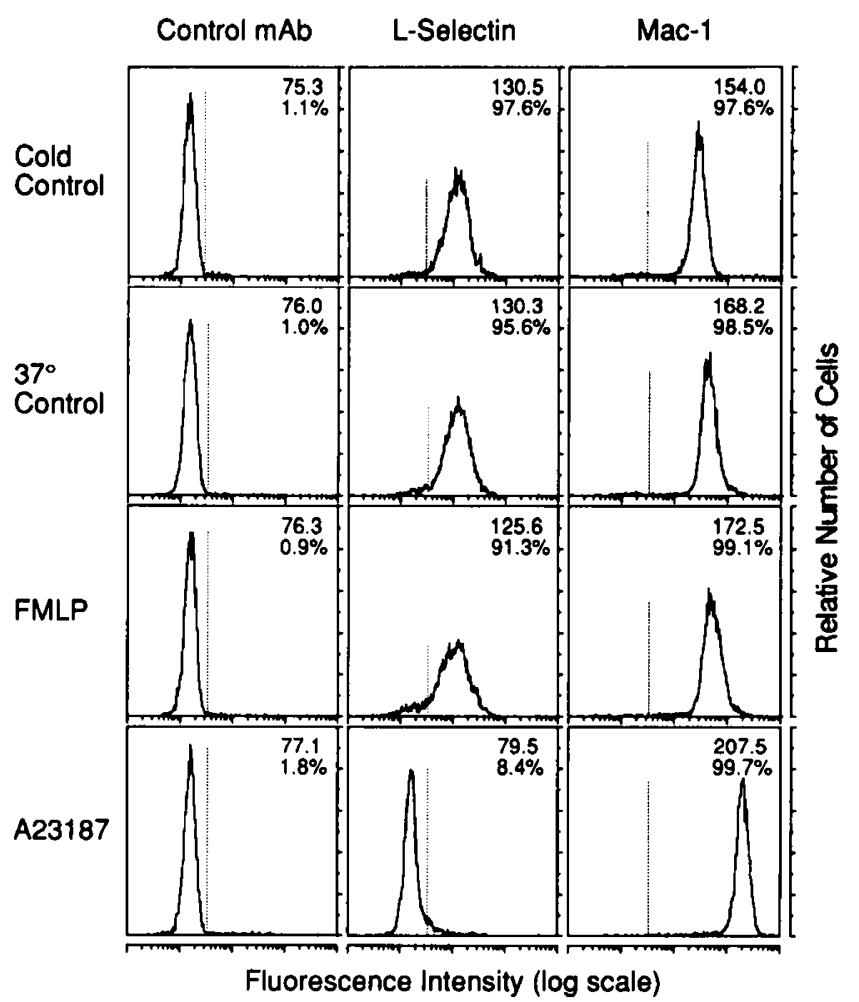

Fig. 1. Representative fluorescence distributions of L-selectin and Mac-1 expression on eosinophils. Aliquots of eosinophils (>99\%) purified from adult peripheral blood were kept on ice (cold control) or incubated

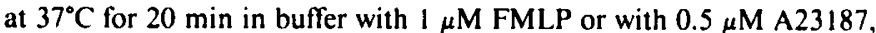
then labeled with PE-conjugated MAb to L-selectin (Leu-8) or the $\alpha$ chain of Mac-1 (Leu-15) or with an isotype-specific control antibody. Each graph shows the relative number of cells at each fluorescence intensity on a 1024-channel logarithmic scale for 4500 total cells. The vertical dotted line indicates the cutoff for positivity defined by the control antibody distribution for each condition. The numbers in the upper right corners indicate the mean fluorescence channel and the percentage of positive cells for the distribution.

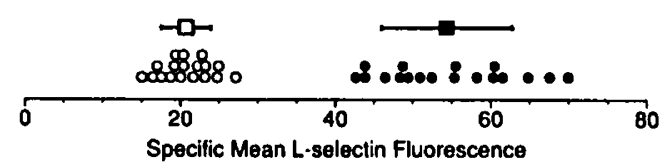

Fig. 2. L-Selectin expression of unstimulated neutrophils (solid symbols) and eosinophils (open symbols) from adult peripheral blood $(n=$ 18). Whole blood leukocytes kept at $0-4^{\circ} \mathrm{C}$ were labeled with the Dreg56 and anti-Leu-1 $1 \mathrm{a} \mathrm{MAb}$ and analyzed by flow cytometry with multiparameter gating as described in Materials and Methods. The squares and error hars indicate the mean \pm SD for each cell type.

under identical conditions. The expression of L-selectin on unstimulated eosinophils and neutrophils from peripheral blood of healthy adult donors is shown in Figure 2. Eosinophils expressed significantly less $L-$ selectin than neutrophils $(20.9 \pm 3.2$ versus $54.5 \pm 8,4 p=0.0001, n=18$ ), in specimens kept at $0-4^{\circ} \mathrm{C}$. Because we had previously found that incubation in buffer for $20 \mathrm{~min}$ at $37^{\circ} \mathrm{C}$ resulted in a $200 \%$ increase in Mac-1 expression on neutrophils and a $60 \%$ increase in Mac-1 expression on eosinophils (41), we examined the effect of warming on L-selectin expression. A $20-\mathrm{min}$ incubation at $37^{\circ} \mathrm{C}$ did not affect the expression of L-selectin on either cell type (eosinophils: $20.9 \pm$ 3.1, neutrophils: $54.5 \pm 7.8 ; n=18$ ), and prolonging the incubation for as long as $60 \mathrm{~min}$ also had no effect.

Kinetics of L-selectin down-regulation. The time course of the changes in eosinophil and neutrophil L-selectin expression induced by stimulation with A23187 were compared in three 
experiments (Fig. 3). For both cell types, there was little change in the first minute after introduction of $A 23187$, then a rapid decrease to $35 \pm 13 \%$ of the initial value for neutrophils and 54 $\pm 9 \%$ for eosinophils at $2 \mathrm{~min}$. At $4 \mathrm{~min}$, neutrophil L-selectin expression was $2.9 \pm 1.3 \%$ of its initial value, compared to 17.6 $\pm 9.3 \%$ for eosinophils $(p=0.05)$, but the overall time courses did not differ significantly. In one experiment, Mac-1 expression was measured in parallel. The time courses of the increases in Mac-1 and the decreases in L-selectin were similar, except that there was no initial delay in the rise of Mac-1 expression.

Effects of chemotactic factors. The responses of eosinophils and neutrophils to increasing concentrations of A23187, PAF, FMLP, and zymosan-activated serum were compared (Fig. 4). Although A23187 stimulation at concentrations of $250 \mathrm{nM}$ or more produced virtually complete losses of L-selectin expression on both cell types, the other substances had much more limited effects on eosinophils compared to neutrophils. PAF at 10- and 100-nM concentrations decreased eosinophil L-selectin expression to $76 \pm 27 \%$ and $59 \pm 27 \%$ of the control value, respectively $(p<0.002)$, compared to $34 \pm 13 \%$ and $17 \pm 7 \%$ for neutrophils $(p<0.0001)$. FMLP at $10 \mathrm{nM}$ had no effect on eosinophils but produced a nearly complete loss of L-selectin expression from neutrophils. FMLP at $1 \mu \mathrm{M}$ reduced eosinophil $\mathrm{L}$-selectin to $84 \%$
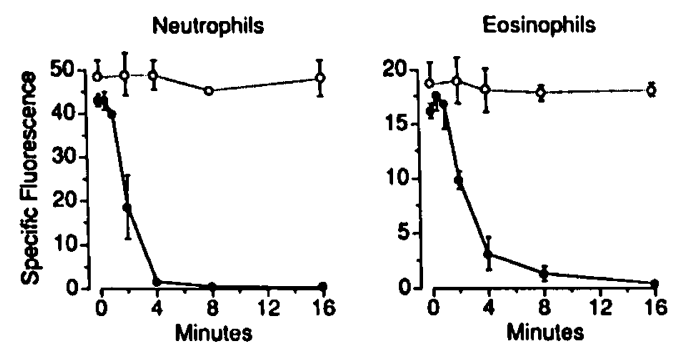

Fig. 3. Kinetics of the changes in eosinophil and neutrophil expression of L-selectin after stimulation. At time zero, buffer containing A23187 at a final concentration of $250 \mathrm{nM}$ (solid circles) or control buffer (open circles) was mixed with whole blood leukocytes at $37^{\circ} \mathrm{C}$. At intervals, aliquots were removed from the $37^{\circ} \mathrm{C}$ bath, cooled by the addition of ice-cold buffer, then labeled with MAb (Dreg-56 or anti-Leu15. plus anti-Leu- 11 a) and analyzed as described in Materials and Methods. The results are the mean \pm SD for experiments with blood from three adults.
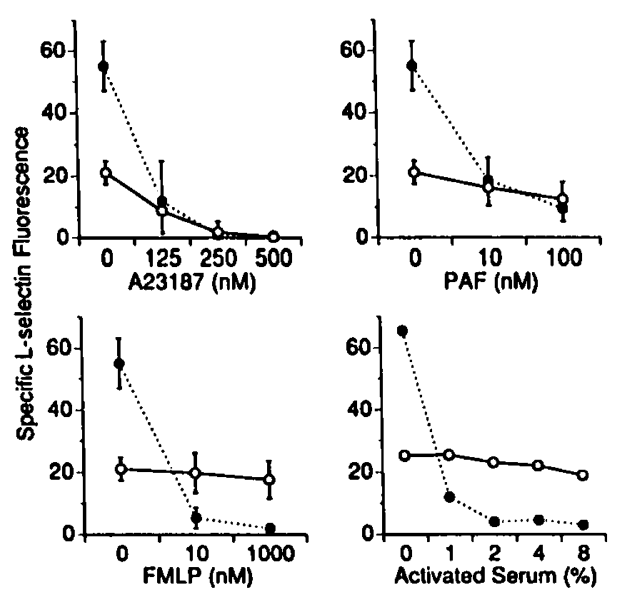

Fig. 4. Dose response of the decrease in L-selectin expression of neutrophils (solid circles) and eosinophils (open circles). Adult whole blood leukocyte suspensions were incubated at $37^{\circ} \mathrm{C}$ for $20 \mathrm{~min}$ with the indicated concentrations of the activating factors, then labeled with Dreg56 and anti-Leu-11a MAb and analyzed as described in Materials and Methods. Results for A23187, PAF, and FMLP are the mean \pm SD for nine experiments. The effect of zymosan-activated autologous serum is shown for one donor. of the control value $(p=0.02)$. Similarly, zymosan-activated serum (Fig. 4), $100 \mathrm{nM}$ recombinant C5a, or $100 \mu \mathrm{M}$ LTB4 each had minimal effects on eosinophil L-selectin while reducing neutrophil L-selectin expression to less than $10 \%$ of the level on unstimulated controls. Incubation with PAF, LTB4, or C5a for $60 \mathrm{~min}$ had no additional effect on either neutrophil or eosinophil L-selectin expression compared to a 20 -min incubation (not shown). In other experiments, histamine $(0.1$ and $1.0 \mathrm{mM})$ and the eosinophil chemotactic peptides Val-Gly-Ser-Glu and AlaGly-Ser-Glu (10 $\mathrm{nM}$ to $100 \mu \mathrm{M})$ had no effect on L-selectin expression of either eosinophils or neutrophils.

Neonatal eosinophils. The use of the CD16 gating method to distinguish eosinophils and neutrophils in cord blood is more demanding than for specimens from healthy adults, because cord blood sometimes contains a significant subpopulation of CD16dim immature myeloid cells with distinctly larger forward light scatter than the eosinophils. We previously showed that this subpopulation was removed when granulocytes were separated by Percoll gradient centrifugation and that, for whole blood, CD16-gating combined with exclusion of this large light-scatter subpopulation gave results for eosinophil Mac-1 expression close to the values obtained for purified eosinophils (41). To confirm the validity of the gating procedure directly, we performed cell sorting experiments (Table 1). For whole blood suspensions from both cord blood and adult specimens, before and after stimulation with A23187, the CD16-dim granulocytes with light scatter in the eosinophil region were $\geq 93 \%$ eosinophils and the CD16bright granulocytes were $\geq 98 \%$ neutrophils. When Percoll-purified granulocytes were sorted, $\geq 98 \%$ of the CD16-dim cells were eosinophils and $>99 \%$ of the CD16-bright cells were neutrophils. For three adult and four neonatal blood samples, we evaluated the possible effects of the cell preparation and analysis methods by comparing the values for $\mathrm{L}$-selectin expression obtained using whole blood with those obtained using Percoll-separated granulocytes. For two adult and two neonatal specimens, these values were also compared with the results for eosinophils purified from the same blood samples. The cell preparation and analysis methods had no significant effects on the results for L-selectin fluorescence. The mean coefficient of variation between pairs of measurements for the same specimens was $\leq 5 \%$ for neutrophils and $\leq 9 \%$ for eosinophils.

L-Selectin expression on unstimulated neutrophils and eosinophils kept at $0-4^{\circ} \mathrm{C}$ was compared for nine pairs of adult and cord blood specimens (Fig. 5). L-Selectin expression was significantly less on neonatal compared to adult eosinophils (mean \pm SD specific fluorescence $10.4 \pm 3.8$ versus $19.4 \pm 2.7, p=$ $0.0001)$ and on neonatal compared to adult neutrophils $(36.4 \pm$ 8.2 versus $55.5 \pm 4.8, p=0.0001$ ). For both adults and neonates, eosinophils expressed significantly less L-selectin than neutrophils $(p=0.0001)$, with a mean ratio of eosinophil to neutrophil L-selectin of $35 \pm 6 \%$ for adults and $29 \pm 8 \%$ for neonates.

It was previously noted that neonatal neutrophils stimulated with FMLP did not appear to down-regulate their expression of L-selectin to the same extent as adult neutrophils (39). In this study, L-selectin down-regulation on neonatal eosinophils and neutrophils stimulated with A23187, FMLP, and PAF was investigated. As with the adult cells, incubation in buffer at $37^{\circ} \mathrm{C}$ did not affect L-selectin expression on either neutrophils or eosinophils. A23187 and PAF produced large reductions in Lselectin on both cell types, whereas FMLP had a much greater effect on neutrophils. When L-selectin expression was expressed as a percentage of its level on the unstimulated controls for each cell (Fig. 6), there were no significant differences in the responses to stimulation of neonatal and adult eosinophils or in the responses of neonatal and adult neutrophils.

\section{DISCUSSION}

The regulation of eosinophil trafficking is likely to involve multiple mechanisms-some that promote the specific exudation 
Table 1. Confirmation of analysis procedure for cord blood by cell sorting*

\begin{tabular}{|c|c|c|}
\hline & $\begin{array}{c}\begin{array}{c}\text { CD16 dim } \\
\text { granulocytes }\end{array} \\
\end{array}$ & $\begin{array}{l}\text { CD16 bright } \\
\text { granulocytes } \\
\end{array}$ \\
\hline & \% Eosinophils & $\%$ Neutrophils \\
\hline $\begin{array}{l}\text { Whole blood }\left(0^{\circ}\right) \\
\text { Adult }\end{array}$ & 95,99 & 99,100 \\
\hline Neonatal & 93,93 & 100,100 \\
\hline $\begin{array}{l}\text { Whole blood (A23187) } \\
\text { Adult }\end{array}$ & 94,97 & 98,98 \\
\hline Neonatal & 97 & 98 \\
\hline $\begin{array}{l}\text { Granulocytes }\left(0^{\circ}\right) \\
\text { Adult }\end{array}$ & 100 & 100 \\
\hline Neonatal & 98 & 100 \\
\hline
\end{tabular}

* Whole blood leukocytes or granulocytes from two adults and two neonates were kept cold $\left(0-4^{\circ}\right)$ or incubated with $500 \mathrm{nM}$ A23187 for 20 min at $37^{\circ} \mathrm{C}$, then labeled with FITC-conjugated anti-Leu-1 la antibody (CD16). Granulocytes were identified by light-scatter gating and sorted into CD16-bright and CD16-dim subpopulations. For the whole-blood preparations, a refined light-scatter gate for the eosinophils was set as described in Materials and Methods. The table shows the number of eosinophils or neutrophils in 100-cell differential counts of Wright-stained slides of the two populations of sorted cells.

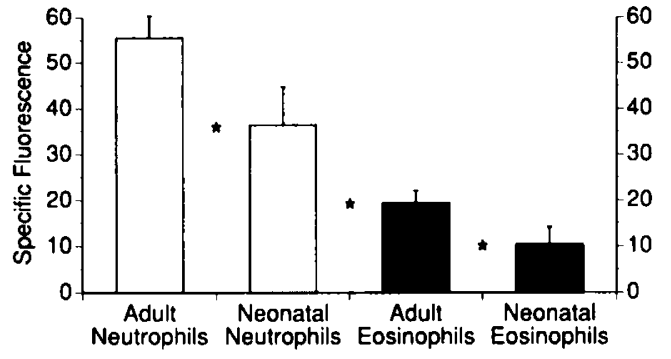

Fig. 5. Expression of L-selectin (mean $\pm \mathrm{SD}$ ) on unstimulated cord blood neutrophils and eosinophils. Whole blood leukocytes or granulocytes from nine pairs of adults and neonates kept at $0-4^{\circ} \mathrm{C}$ were labeled with Dreg-56 and anti-Leu-11a MAb and analyzed as described in Materials and Methods. ${ }^{*}, p=0.0001$ between groups.

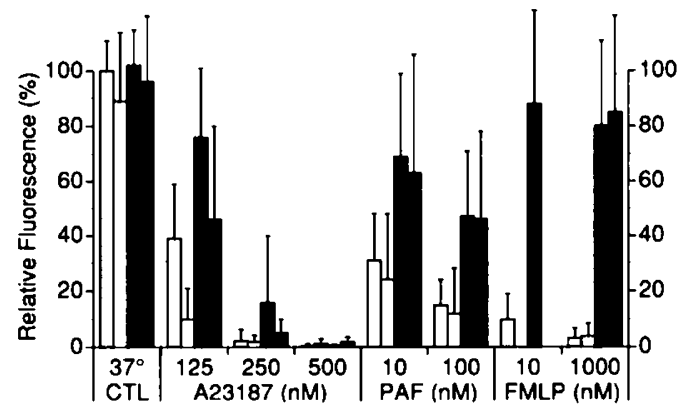

Fig. 6. Comparison of the responses of adult neutrophils (light shad. ing), neonatal neutrophils (white), adult eosinophils (dark shading), and neonatal eosinophils (black) to incubation with activating factors. Whole blood leukocytes were kept cold or incubated for $20 \mathrm{~min}$ at $37^{\circ} \mathrm{C}$ in buffer $\left(37^{\circ}\right.$ control) or with the indicated concentrations of A23187, PAF, and FMLP, then labeled with Dreg-56 and anti-Leu-11a MAb and analyzed as described in Materials and Methods. L-Selectin fluorescence was expressed as a percentage of the cold control value for each donor. The graph shows the results (mean $\pm S D$ ) for five pairs of adults and neonates.

of eosinophils, and others that normally limit their involvement at sites of infection or inflammation. The results of this study suggest two possible mechanisms that may tend to limit eosinophil exudation: diminished quantitative expression of L-selectin on eosinophils compared to neutrophils and a diminished responsiveness of eosinophils to chemotactic factors.

We found that the baseline expression of $\mathrm{L}$-selectin on unstimulated peripheral blood eosinophils from healthy adults was about one third the level expressed on neutrophils in the same specimens. It was shown previously that under conditions of flow corresponding to the shear stress prevailing in postcapillary venules, neutrophil adherence to IL-1-stimulated endothelium was dependent on L-selectin and virtually independent of CD18dependent mechanisms (32). Furthermore, there was a direct correlation between the quantitative expression of L-selectin and the number of adherent cells (32). It is possible, therefore, that the reduced expression of $\mathrm{L}$-selectin on eosinophils may result in diminished endothelial adhesion of eosinophils compared to neutrophils in conditions where L-selectin-mediated adhesion may be important. At present, there is no information on the relative importance of different adhesion mechanisms for eosinophils under conditions of flow.

The second mechanism that may serve to limit exudation of eosinophils is their relative unresponsiveness to direct stimulation with chemotactic factors. We found that, although FMLP, C5a, PAF, and LTB4 all induced large changes in neutrophil Lselectin expression, eosinophils in the same cell suspensions had a modest response to PAF and minimal or no responses to C5a, FMLP, and LTB4. The magnitudes of the eosinophil L-selectin responses to these factors followed the pattern previously found for Mac-1 up-regulation on eosinophils (41). These results suggest that some additional priming or activation signal is required to initiate a response to $\mathrm{C5a}$ in eosinophils, because PAF and C5a are both potent chemotactic factors for eosinophils $(49,50)$. It should be noted that the processes involved in triggering eosinophil migration are probably complex and that, although the precise roles of these particular adhesion receptor responses are not known, they are likely to be coordinated with other mechanisms. The idea that the diminished L-selectin and Mac-1 responses to direct stimulation with chemotactic factors are important characteristics of eosinophils is therefore supported by the results of Giembycz et al. (51), who found that PAF was more effective than C5a, FMLP, and phorbol myristate acetate in stimulating release of thromboxane $B_{2}$ by eosinophils but far less effective than A23187. The mechanisms involved in eosinophil activation or lack of activation by specific chemotactic factors are not well understood, except that eosinophils lack a high-affinity receptor for FMLP $(44,47,48)$. In addition, the eosinophil C5a receptor has been shown to be distinct from the neutrophil C5a receptor (52). Elucidation of the mechanisms responsible for the differing responses of neutrophils and eosinophils to chemotactic stimulation will be an important goal for future studies.

These studies demonstrate that down-regulation of L-selectin expression on human eosinophils was coordinated with Mac-1 up-regulation, as previously described for neutrophils $(33,34)$. There were two aspects to this coordination. First, the changes in Mac-1 and L-selectin were correlated in time, proceeding to 
completion within 4 to $8 \mathrm{~min}$, and the kinetics for eosinophils and neutrophils were similar. Second, the magnitude of the changes in eosinophil Mac-1 and L-selectin induced by a particular stimulus were similar, i.e. the stimulus triggered both processes to the same relative extent. However, some differences in the timing and initiation of the L-selectin and Mac-1 responses were observed. After exposure of the cells to A23187, Mac-1 expression began to increase immediately, whereas there was a 1 - min lag before $\mathrm{L}$-selectin decreased. In addition, Mac-1 continued to rise after the changes in L-selectin were complete. We also found that a $20-$ min incubation at $37^{\circ} \mathrm{C}$ in buffer produced no change in either eosinophil or neutrophil L-selectin, whereas Mac-1 increased significantly. This shows that the Mac-1 and Lselectin responses can be dissociated in part. Future studies of signal transduction pathways in neutrophils and eosinophils may be able to exploit this observation.

The cell sorting studies reported here and the demonstration of the lack of an effect of the cell preparation method on eosinophil and neutrophil expression of L-selectin add to the previous description of the use of CD16 to distinguish eosinophils and neutrophils in mixed suspensions of neonatal leukocytes (41). Back-gating of light scatter allowed us to distinguish eosinophils from the CDI6-dim immature myeloid cells that were present in cord blood but not in adult peripheral blood. Alternatively, the complication introduced by this population could be avoided by partial purification on a simple two-layer Percoll gradient. For cell populations with a very large number of immature myeloid cells (e.g. bone marrow) or where extensive in vivo loss of CD16 on neutrophils may occur (53), the ability of light scatter to discriminate eosinophils from other CD16-dim cells would need to be specifically examined.

Newborn infants have an enhanced susceptibility to serious bacterial infections, which may be due in part to transient abnormalities of neutrophil function. Studies of neonatal animals have demonstrated diminished or delayed emigration of neutrophils into sites of inflammation, and numerous in vitro studies of human neutrophils have found defects in chemotactic functions $(54,55)$. Although the etiology of these changes is not fully understood, neonatal neutrophils have been shown to have specific abnormalities of adhesion and transendothelial migration related to L-selectin and Mac-1 $(39,55)$. The results for neonatal neutrophils in this study confirmed the previous finding of significantly reduced expression of L-selectin compared to adult neutrophils (39). However, in that study the magnitude of the reduction was greater, and stimulation with FMLP failed to induce a further decrease of L-selectin on neonatal neutrophils. We attribute these differences to the use in the previous study of neutrophils isolated at room temperature by dextran sedimentation and Ficoll-Hypaque centrifugation. In the present study, we found that neonatal neutrophils as well as neonatal eosinophils responded to FMLP and other factors to the same relative extent as the adult cells.

This study shows that neonatal eosinophils have decreased expression of L-selectin compared to peripheral blood eosinophils from normal adults. The ratio of neonatal to adult eosinophil Lselectin expression (54\%) was similar in magnitude to the ratio for neonatal to adult neutrophils $(66 \%)$. We previously demonstrated that maximum expression of Mac- 1 on neonatal eosinophils in response to stimulation was $68 \%$ of that of adult eosinophils, compared to $67 \%$ for neonatal versus adult neutrophils (41). This remarkable parallelism suggests that a common mechanism may underlie the abnormal expression of these receptors on both eosinophils and neutrophils of neonates.

Furthermore, this parallelism raises the possibility that neonatal eosinophils may have Mac-1 and L-selectin-related abnormalities in adhesion and migration similar to those of neonatal neutrophils. For neonatal and adult neutrophils, expression of $\mathrm{L}-$ selectin correlated with adhesion of neutrophils to IL-1-stimulated endothelium under conditions of flow where CD18-dependent mechanisms were unimportant (39). It is not presently known whether L-selectin-mediated adhesion has a similar importance for eosinophils under these conditions. It is also not known whether there is a direct relationship between the Mac1-related abnormalities in adhesion and transendothelial migration of neonatal neutrophils and their abnormality of quantitative expression, because abnormalities of activation of Mac-1 are also thought to contribute (55).

Future study of neonatal eosinophils together with neonatal neutrophils may provide a useful physiologic model for investigating the specific functions of Mac-1, L-selectin, and other adhesion receptors. If eosinophils prove to have functional defects in Mac-1 and L-selectin similar to those of neonatal neutrophils $(39,55)$, the increased involvement of eosinophils in neonatal inflammatory exudates would require a significant enhancement of other adhesion systems, such as the VLA-4/ VCAM-1 interaction (21-24), or other mechanisms entirely. We conclude that neonatal eosinophils have abnormalities of $\mathrm{L}$ selectin expression similar to neonatal neutrophils and that decreased expression of L-selectin and a diminished responsiveness to chemotactic factor stimulation are possible mechanisms that may tend to limit the exudation of eosinophils. The adhesion and migration properties of neonatal eosinophils will be important areas for further study.

Acknowledgments. The authors thank Ingrid Schmidt and Valentin Isacescu, M.D., of the Flow Cytometry Core Laboratory, Johnson Comprehensive Cancer Center, UCLA, for expert assistance.

\section{REFERENCES}

1. Gleich GJ 1990 The eosinophil and bronchial asthma: current understanding. J Allergy Clin Immunol 8:422-436

2. Hoidal JR 1990 The eosinophil and acute lung injury. Am Rev Respir Dis 142:1245-1246

3. Weller PF 1991 The immunobiology of eosinophils. N Engl J Med 324: 1110-1118

4. Eitzman DV, Smith RT 1959 The nonspecific inflammatory cycle in the neonatal infant. Am J Dis Child 97:326-334

5. Bullock JD, Robertson AF, Bodenbender JG, Kontras SB, Miller CE 1969 Inflammatory response in the neonate re-examined. Pediatrics 44:58-61

6. Roberts RL, Ank BJ, Salusky IB, Stiehm ER 1990 Purification and properties of peritoneal eosinophils from pediatric dialysis patients. J Immunol Methods 126:205-211

7. Harris JR, Schick B 1956 Erythema neonatorum. Am J Dis Child 92:27-33

8. Gibson EL, Vaucher Y, Corrigan JJ 1979 Eosinophilia in premature infants: relationship to weight gain. J Pediatr 95:99-101

9. Bhat AM, Scanlon JW 1981 The pattern of eosinophilia in premature infants J Pediatr 98:612-616

10. Smith JB, Campbell DE, Ludomirsky A, Polin RA, Douglas SD, Garty BZ, Harris MC 1990 Expression of the complement receptors CR I and CR 3 and the type III Fcy receptor on neutrophils from newborn infants and from fetuses with Rh disease. Pediatr Res 28:120-126

11. Forestier F. Daffos F. Galactéros F, Bardakjian J, Rainaut M, Beuzard Y 1986 Hematological values of 163 normal fetuses between 18 and 30 weeks of gestation. Pediatr Res 20:342-346

12. Carlos TM, Harlan JM 1990 Membrane proteins involved in phagocyte adherence to endothelium. Immunol Rev 114:5-28

13. Springer TA 1990 Adhesion receptors of the immune system. Nature 356: 425-434

14. Wegner CD. Smith CW, Rothlein R 1988 CD18 dependence of primate eosinophil adherence in vitro. Springer-Verlag. Titisee, Germany, pp 208214

15. Lamas AM. Mulroney CM. Schleimer RP 1988 Studies on the adhesive interaction between purified eosinophils and cultured vascular endothelial cells. J Immunol 140:1500-1505

16. Kimani G, Tonnesen MG, Henson PM 1988 Stimulation of eosinophil adherence to human vascular endothelial cells in vitro by platelet-activating factor. J Immunol 140:3161-3166

17. Walsh GM, Hartnell A, Wardlaw AJ, Kurihara K, Sanderson CJ, Kay AB 1990 IL-5 enhances the in vitro adhesion of human esinophils, but not neutrophils, in a leucocyte integrin (CDI1/18)-dependent manner. Immunology 71:258-265

18. Anderson DC. Springer TA 1987 Leukocyte adhesion deficiency: an inherited defect in the Mac-1, LFA-1, and p150.95 glycoproteins. Annu Rev Med 38: 175-194

19. Anderson DC, Schmalsteig FC, Finegold MJ. Hughes BJ, Rothlein R, Miller LJ, Kohl S, Tosi MF, Jacobs RL, Waldrop TC, Goldman AS. Shearer WT. Springer TA 1985 The severe and moderate phenotypes of heritable Mac-1, LFA-I deficiency: their quantitative definition and relation to leukocyte dysfunction and clinical features. J Infect Dis 152:668-689 
20. Kyan-Aung U, Haskard DO, Poston RN, Thornhill MH, Lee TH 1991 Endothelial leukocyte adhesion molecule- 1 and intercellular adhesion molecule-1 mediate the adhesion of eosinophils to endothelial cells in vitro and are expressed by endothelium in allergic cutaneous inflammation in vivo. J Immunol 146:521-528

21. Bochner BS, Luscinskas FW, Gimbrone Jr MA, Newman W, Sterbinsky SA, Derse-Anthony CP, Klunk D, Schleimer RP 1991 Adhesion of human basophils, eosinophils, and neutrophils to interleukin-1-activated human vascular endothelial cells: contributions of endothelial cell adhesion molecules. J Exp Med 173:1553-1556

22. Weller PF, Rand TH, Goelz SE, Chi-Rosso G, Lobb RR 1991 Human eosinophil adherence to vascular endothelium mediated by binding to vascular cell adhesion molecule 1 and endothelial leukocyte adhesion molecule 1. Proc Natl Acad Sci USA 88:7430-7433

23. Walsh GM, Mermod JJ, Hartnell A, Kay AB, Wardlaw AJ 1991 Human eosinophil, but not neutrophil, adherence to IL-I-stimulated human umbilical vascular endothelial cells is $\alpha_{4} \beta_{1}$ (very late antigen-4) dependent. J Immunol 146:3419-3423

24. Dobrina A, Menegazzi R, Carlos TM, Nardon E, Cramer R, Zacchi T, Harlan JM. Patriarca P 1991 Mechanisms of eosinophil adherence to cultured vascular endothelial cells: eosinophils bind to the cytokine-induced endothelial ligand vascular cell adhesion molecule-1 via the very late activation antigen-4 integrin receptor. J Clin Invest 88:20-26

25. Bevilacqua $M$, Butcher E, Furie Ba., Furie Br., Gallatin M. Gimbrone $M$, Harlan J, Kishimoto K, Lasky L, McEver R, Paulson J, Rosen S, Seed B, Siegelman M, Springer T, Stoolman L, Tedder T, Varki A, Wagner D, Weissman 1, Zimmerman G 1991 Selectins: a family of adhesion receptors Cell 67:233

26. Stoolman LM 1989 Adhesion molecules controlling lymphocyte migration. Cell 56:907-910

27. Lewinsohn DM, Bargatze RF, Butcher EC 1987 Leukocyte-endothelial cell recognition: evidence of a common molecular mechanism shared by neutrophils, lymphocytes, and other leukocytes. J Immunol 138:4313-432 I

28. Camerini D, James SP, Stamenkovic I. Seed B 1989 Leu-8/TQ1 is the human equivalent of the Mel-14 lymph node homing receptor. Nature 342:78-82

29. Tedder TF, Penta AC, Levine HB, Freedman AS 1990 Expression of the human leukocyte adhesion molecule, LAMI: identity with the TQ1 and Leu-8 differentiation antigens. J Immunol 144:532-540

30. Ord DC, Ernst TJ. Zhou LJ. Rambaldi A, Spertini O, Griffin J, Tedder TF 1990 Structure of gene encoding the human leukocyte adhesion molecule-1 (TQ1, Leu-8) of lymphocytes and neutrophils. J Biol Chem 265:7760-7767

31. Kishimoto TK, Jutila MA. Butcher EC 1990 Identification of a human peripheral lymph node homing receptor: a rapidly down-regulated adhesion molecule. Proc Natl Acad Sci USA 87:2244-2248

32. Smith CW, Kishimoto TK, Abbass O, Hughes B, Rothlein R, McIntire LV Butcher E, Anderson DC 1991 Chemotactic factors regulate lectin adhesion molecule I (LECAM-1)-dependent neutrophil adhesion to cytokine-stimulated endothelial cells in vitro. J Clin Invest 87:609-618

33. Kishimoto TK, Jutila MA, Berg EL, Butcher EC 1989 Neutrophil Mac-1 and MEL-14 adhesion proteins inversely regulated by chemotactic factors. Science 245:1238-1241

34. Jutila MA, Kishimoto TK, Butcher EC 1990 Regulation and lectin activity of the human neutrophil peripheral lymph node homing receptor. Blood 76 $178-183$

35. Berg M, James SP 1990 Human neutrophils release the Leu-8 lymph node homing receptor during cell activation. Blood 76:2381-2388

36. Arnaout MA 1990 Structure and function of the leukocyte adhesion molecules CD11/CD18. Blood 75:1037-1050

37. Spertini O, Kansas GS, Munro JM, Griffin JD, Tedder TF 1991 Regulation of leukocyte migration by activation of the leukocyte adhesion molecule-1 (LAM-1) selectin. Nature 349:691-694

38. Jutila MA. Rott L, Berg EL, Butcher EC 1989 Function and regulation of the neutrophil MEL-14 antigen in vivo: comparison with LFA-1 and Mac-1.J Immunol 143:3318-3324

39. Anderson DC, Abbassi O, Kishimoto TK, Koenig JM, McIntire LV, Smith CW 1991 Diminished lectin-, epidermal growth factor-, complement binding domain-cell adhesion molecule-1 on neonatal neutrophils underlies their impaired CDI8-independent adhesion to endothelial cells in vitro. J Immunol 146:3372-3379

40. Griffin JD, Spertini O, Ernst TJ, Belvin MP, Levine HB, Kanakura Y, Tedder TF 1990 Granulocyte-macrophage colony-stimulating factor and other cytokines regulate surface expression of the leukocyte adhesion molecule-1 on human neutrophils, monocytes, and their precursors. J Immunol 145: 576-584

41. Smith JB, Kunjummen RD, Raghavender BH 1991 Eosinophils and neutrophils of human neonates have similar impairments of quantitative upregulation of Mac-1 (CDI lb/CD18) expression in vitro. Pediatr Res 30: 355-361

42. Anderson DC, Freeman KLB, Heerdt B, Hughes BJ, Jack RM, Smith CW 1987 Abnormal stimulated adherence of neonatal granulocytes: impaired induction of surface MAC-1 by chemotactic factors or secretagogues. Blood 70:740-750

43. Bruce MC, Baley JE, Medvik KA, Berger M 1987 Impaired surface membrane expression of $\mathrm{C} 3 \mathrm{bi}$ but not $\mathrm{C} 3 \mathrm{~b}$ receptors on neonatal neutrophils. Pediatr Res 21:306-311

44. Roberts RL, Gallin JI 1985 Rapid method for isolation of normal human peripheral blood eosinophils on discontinuous Percoll gradients and comparison with neutrophils. Blood 65:433-440

45. Hansel TT, Pound JD, Pilling D, Kitas GD, Salmon M, Gentle TA, Lee SS, Thompson RA 1989 Purification of human blood eosinophils by negative selection using immunomagnetic beads. J Immunol Methods 122:97-103

46. Terstappen LW, Hollander Z, Meiners H, Loken MR 1990 Quantitative comparison of myeloid antigens on five lineages of mature peripheral blood cells. J Leukocyte Biol 48:138-148

47. Yazdanbakhsh M. Eckmann CM, Koenderman L, Verhoeven AJ, Roos D 1987 Eosinophils do respond to fMLP. Blood 70:379-383

48. Koenderman L, Kok PTM, Hamelink ML, Verhoeven AJ,Bruijnzeel PLB 1988 An improved method for the isolation of eosinophilic granulocytes from peripheral blood of normal individuals. $J$ Leukocyte Biol 44:79-86

49. Wardlaw AJ, Mogbel R, Cromwell O, Kay AB 1986 Platelet activating factor: a potent chemotactic and chemokinetic factor for human eosinophils. J Clin Invest 78:1701-1706

50. Morita E, Schröder J-M, Christophers E 1989 Differential sensitivities of purified human eosinophils and neutrophils to defined chemotaxins. Scand J Immunol 29:709-716

51. Giembycz MA, Kroegel C. Barnes PJ 1990 Platelet activating factor stimulates cyclo-oxygenase activity in guinea pig eosinophils. J Immunol 144 3489-3497

52. Gerard NP, Hodges MK, Drazen JM, Weller PF, Gerard C 1989 Characterization of a receptor for C5a anaphylatoxin on human eosinophils. J Biol Chem 264:1760-1766

53. Boros P, Gardos E, Bekesi GJ, Unkeless JC 1990 Change in expression of $F_{c} \gamma \mathbf{R}_{\text {III }}(\mathrm{CD} 16)$ on neutrophils from human immunodeficiency virus-infected individuals. Clin Immunol Immunopathol 54:281-289

54. Hill HR 1987 Biochemical, structural, and functional abnormalities of polymorphonuclear leukocytes in the neonate. Pediatr Res 22:375-382

55. Anderson DC, Rothlein R, Marlin SD, Krater SS, Smith CW 1990 Impaired transendothelial migration by neonatal neutrophils: abnormalities of Mac-1 (CD11b/CD18)-dependent adherence reactions. Blood 76:2613-2621 